\title{
Review Article \\ Cognitive Impairment in Chronic Kidney Disease: Vascular Milieu and the Potential Therapeutic Role of Exercise
}

\author{
Ulf G. Bronas, Houry Puzantian, and Mary Hannan \\ College of Nursing, Department of Biobehavioral Health Science, University of Illinois at Chicago, Chicago, IL, USA \\ Correspondence should be addressed to Ulf G. Bronas; bronas@uic.edu
}

Received 27 November 2016; Accepted 28 February 2017; Published 19 April 2017

Academic Editor: Hiroyuki Shimada

Copyright (C) 2017 Ulf G. Bronas et al. This is an open access article distributed under the Creative Commons Attribution License, which permits unrestricted use, distribution, and reproduction in any medium, provided the original work is properly cited.

\begin{abstract}
Chronic kidney disease (CKD) is considered a model of accelerated aging. More specifically, CKD leads to reduced physical functioning and increased frailty, increased vascular dysfunction, vascular calcification and arterial stiffness, high levels of systemic inflammation, and oxidative stress, as well as increased cognitive impairment. Increasing evidence suggests that the cognitive impairment associated with CKD may be related to cerebral small vessel disease and overall impairment in white matter integrity. The triad of poor physical function, vascular dysfunction, and cognitive impairment places patients living with CKD at an increased risk for loss of independence, poor health-related quality of life, morbidity, and mortality. The purpose of this review is to discuss the available evidence of cerebrovascular-renal axis and its interconnection with early and accelerated cognitive impairment in patients with CKD and the plausible role of exercise as a therapeutic modality. Understanding the cerebrovascular-renal axis pathophysiological link and its interconnection with physical function is important for clinicians in order to minimize the risk of loss of independence and improve quality of life in patients with CKD.
\end{abstract}

\section{Introduction}

Chronic kidney disease (CKD) affects $45 \%$ of adults older than 70 years of age in the US [1]. The incidence of CKD will increase significantly over the next decade due to the increasing incidence of diabetes and hypertension in the rapidly aging US population. The economic cost of CKD is staggering with Medicare spending for patients with CKD aged 65 and older exceeding $\$ 50$ billion in 2013, representing $20 \%$ of all Medicare spending in this age group [2]. Contributing to the high cost of CKD is the remarkably high prevalence of cognitive impairment or overt dementia that ranges $20-50 \%$ in older patients with moderate CKD [38] and may reach as high as $70 \%$ in severe $\mathrm{CKD} /$ dialysis [9]. Cognitive impairment impacts patients negatively by contributing to functional dependence and behavioral symptoms that result in poor outcomes and decreased medication and medical care compliance. These negative consequences result in a downward spiral of functional decline and an accelerated loss of independence, which leads to premature institutionalization [10-16]. The negative impact of cognitive impairment on quality of life and emotional wellbeing is significant, and it even affects employment rates negatively [17-20]. Moreover, cognitive function for incident dialysis patients has been found to be correlated with frailty and measures of depression [21]. Additionally, it more than doubles mortality risk and increases days spent in the hospital $[15,22]$, contributing to the tremendous individual, societal, and economical burden of CKD. We will review the vascular milieu as it is associated with cognitive decline in patients with kidney disease and the potential therapeutic role of exercise.

\section{Measurement of Cognitive Impairment}

Cognitive impairment is commonly referred to as a reduction in global cognition that is new and affects at least 2 areas of cognitive function that can be measured using a standard cognitive function test (e.g., Mini Mental State Exam (MMSE) or the Montreal Cognitive Assessment (MOCA)) $[23,24]$. Impairment can be evident in various cognitive domains: executive function (judgement and planning), language, attention, memory, and visual-spatial learning. Mild cognitive impairment (MCI) is commonly defined as a deficit 
in global cognition that is not consistent with aging and has not progressed to overt dementia. Although there is a lack of a consensus for a standard definition of MCI, it is known to be present with a performance of 1.5-1.99 standard deviations below the standard norm on a given cognitive test. MCI is mostly manifested in short-term memory loss but can also be manifested as impaired language and executive functions $[25,26]$. Importantly, progression from MCI to overt dementia is approximately $15 \%$ per year in older patients [26]. Dementia on the other hand is used as the umbrella term for moderate/severe progressive cognitive impairment often defined as scoring 2 standard deviations below population norms in at least 2 cognitive domains [27]. Importantly, overt dementia leads to a loss of independent daily function whereas MCI does not appear to significantly affect independent daily function.

\section{Cognitive Impairment and Dementia in CKD}

It is well established that patients with kidney disease commonly have some degree of cognitive impairment and that kidney dysfunction is associated with a more rapid decline in mental function than in age matched comparisons $[28,29]$. As many as $20-50 \%$ of patients with moderate CKD have established cognitive impairment or overt dementia [3-8, 30]. It should be noted that the actual population prevalence and incidence of cognitive impairment are likely underreported because published studies are primarily clinic-based and not true population studies. The United States Renal Data System Annual Data Report found a lower prevalence of cognitive impairment in CKD patients (7.6-16.8\%) [22]. However, the true population prevalence is likely substantially higher. This is evidenced by Kurella et al. [6] who reported a 23-28\% prevalence of cognitive impairment in stages 3-4 CKD patients $(n=80)$ seen in clinical practice and Murray et al. [9] who reported a prevalence of MCI or dementia in $87 \%$ of older dialysis patients. Most published studies have reported a prevalence of cognitive impairment of $20-50 \%$ in CKD patients and up to $70 \%$ in older patients on dialysis [39]. Unfortunately, less than $5 \%$ of all renal disease patients with cognitive impairment have been screened or received a medical diagnosis $[9,31]$. This suggests that cognitive impairment in this group of patients is severely underdetected and not adequately addressed.

The degree of renal dysfunction appears to be correlated with the degree of cognitive impairment. Cognitive impartment has been shown in multiple studies to be associated with deteriorating renal function well before requiring dialysis, although this association is particularly strong in patients requiring dialysis [31]. Increased serum cystatin $\mathrm{C}$ and albuminuria are also associated with accelerated cognitive decline [32-34]. Studies have shown a $15-25 \%$ increased risk of cognitive impairment for every $10 \mathrm{ml} / \mathrm{min}$ per $1.73 \mathrm{~m}^{2}$ reduction in the estimated glomerular filtration rate (eGFR). Further, there is an increased odds ratio of 2.43 (95\% CI 1.38 to 4.29 ) for cognitive impairment in patients with an eGFR of $<45$ $\mathrm{ml} / \mathrm{min} /$ per $1.73 \mathrm{~m}^{2}$ even after adjustment for confounders [ 7 , 35]. Thus, patients with CKD appear to have at least a twofold increased risk of cognitive impairment than those without CKD $[7,8,30]$. This risk increases to fourfold with further reductions in eGFR to $<30 \mathrm{ml} / \mathrm{min}$ per $1.73 \mathrm{~m}^{2}$ independent of potential confounders $[8,35]$. These findings translate to patients with CKD having an increased and accelerated risk of cognitive aging equivalent to 3.6-7 years compared to the general population $[32,36]$. However, current physical examination and medical history for patients with CKD or endstage renal disease (ESRD) do not include cognitive function measures.

In terms of the clinical implications of cognitive impairment in CKD, improving support and access to psychology and social professionals, support groups, and patient education are likely to improve outcomes, although this has yet to be determined. Support of patients with CKD should also include counseling with pharmacists and providers regarding the risk of polypharmacy and potential interactions with patient-initiated supplements. The prevalence of cognitive impairment and dementia in the growing CKD population is likely to cause strain on the healthcare system, individuals, and family members. It is imperative that clinicians recognize the risk of cognitive impairment in the CKD population and include screening for cognitive impairment and initiate prompt treatment and coping strategies.

\section{Brain Structure in Renal Disease}

Magnetic resonance imaging (MRI) techniques have been used to assess brain structure and function in patients with CKD. Older MRI techniques have shown general cerebral atrophy of the hippocampus, cortical atrophy, and prominent lesions of the frontal lobes [37-39]. More recent MRI studies have been able to show deterioration of functional structures including reduced deep white matter volume, white matter hyperintensities representing small vessel disease, white matter lesions, and overt white matter disease [4044]. Moreover, white matter lesions (degeneration of cells in the white matter) are frequent (up to $70 \%$ in dialysis patients) in CKD patients, even before requiring dialysis, suggesting that structural alterations begin early in the CKD disease process [40-44]. White matter lesions likely reflect vascular damage and cerebral ischemic areas. Advanced MRI techniques including diffusion tensor imaging (DTI) have shown subtle alterations in brain structural connectivity of the white matter via mean diffusivity (MD) and fractional anisotropy (FA). The white matter is important for coordinating interactions between different regions of the brain and is essential for normal functioning of the brain [4549]. Impaired white matter integrity appears to be a primary contributor to cognitive decline in CKD and is strongly affected by the internal vascular milieu [45, 46]. Several studies have reported a correlation between MD and FA values and neuropsychiatric testing for patients with CKD, on hemodialysis, and after transplant [50-52]. The use of advanced MRI measures such as DTI may provide a method to diagnose early risk of cognitive decline before symptom presentation [53]. Moreover, several newer MRI techniques 
are emerging such as multicomponent relaxometry techniques that may provide a tool to understand the etiology and the impact of risk factor contribution to cognitive decline in patients with renal disease [47, 50-55]. Notably, structural and functional brain changes appear to occur in conjunction with reduced cerebral blood flow, likely related to systemic and cerebral endothelial dysfunction and arterial calcification [45, 56, 57]. Interestingly, Zhang et al. (2016) attempted to evaluate potential changes in white matter integrity in a small nonrandomized single arm study by assessing patients' brain functional connectivity before and after kidney transplantation [55]. They reported that structural connectivity values were abnormal before transplantation but returned close to normal values one-month after transplantation. Radić et al. (2011) observed improvement in cognitive function following transplantation, which was maintained at 2-year followup [58]. The reasons for these findings are not clear and need to be confirmed in appropriately powered randomized, controlled trials. However, these studies are encouraging and suggest that the adverse brain structural changes may be susceptible to reversal although it is clear that much additional research is needed before any conclusions can be made.

\section{Etiology of Cognitive Decline in Kidney Disease}

The most common type of dementia in the general population is neurodegenerative dementia (as seen in Alzheimer's disease) often manifested as atrophy of the hippocampus. Patients with renal disease are more likely to have large and small blood vessel disease, which causes white matter disease and reduced white matter integrity related cognitive impairment that often is superimposed on neurodegenerative disease. This vascular disease results in a high rate and susceptibility of cerebrovascular disease including subclinical microvascular cerebral disease and overt stroke [59-62]. Cerebral microbleeds occur in up to $60 \%$ of all patients with CKD and appear to be more frequent in patients with black ethnicity $[63,64]$. Moreover, CKD patients have a fivefold increased risk of developing clinical and subclinical cerebrovascular disease, and the annual incidence of stroke is approximately $10 \%$, compared to $2.5 \%$ in an age and sex matched population without CKD [22, 65]. This rate is even higher in the dialysis population and may reach as high as a tenfold increased incidence of stroke compared to the general population $[66,67]$. There is therefore a strong likelihood that patients with CKD are at an increased risk for cognitive impairment due to vascular disease-related causes, manifested as cerebral microinfarcts and white matter disease, and not overt Alzheimer's disease per se [68]. The cerebral vascular disease appears to act in conjunction with a neurodegenerative disease process mediated in part by uremic toxins, creatinine levels, and even cystatin $C$ levels [62]. It should be noted that the pathophysiology and etiology of cognitive decline in CKD are complex and multifaceted, and far from completely understood.

\section{Risk Factors Associated with Cognitive Decline in CKD}

Risk factors for cognitive decline in patients with CKD are listed as follows:

Demographic Factors

African American

Hispanic

Female sex

Older age

Low education

Clinical Factors

Hypertension

Diabetes

Dyslipidemias

Polypharmacy

Sleep quality

Depression

Vascular Milieu

Oxidative stress

Inflammation

Hyperhomocysteinemia

Uremia

Albuminuria

Dialysis Procedure Specific Risk Factors for Cognitive Impairment in End-Stage Renal Disease
Volume and electrolyte fluctuation
Cerebral edema
Cerebral hypoperfusion
Hypotension during dialysis
Excessive cytokine release
Microembolism
Delirium

The traditional risk factors for cerebrovascular disease include African American and Hispanic ethnicity, dyslipidemia, hypertension, diabetes mellitus, female sex, education status, and older age [7, 31, 35, 69-75]. Vascular risk factors will be discussed in detail below. Various clinical factors that are unique to the CKD population contribute to cognitive impairment. These include a high rate of undiagnosed depression and polypharmacy-related side effects or interactions $[19,20]$. Patients with renal disease often have significant fatigue and daytime sleepiness related to poor sleep quality, which could contribute to further cognitive decline [76]. It should be noted that patients undergoing hemodialysis have many additional risk factors predisposing them to 


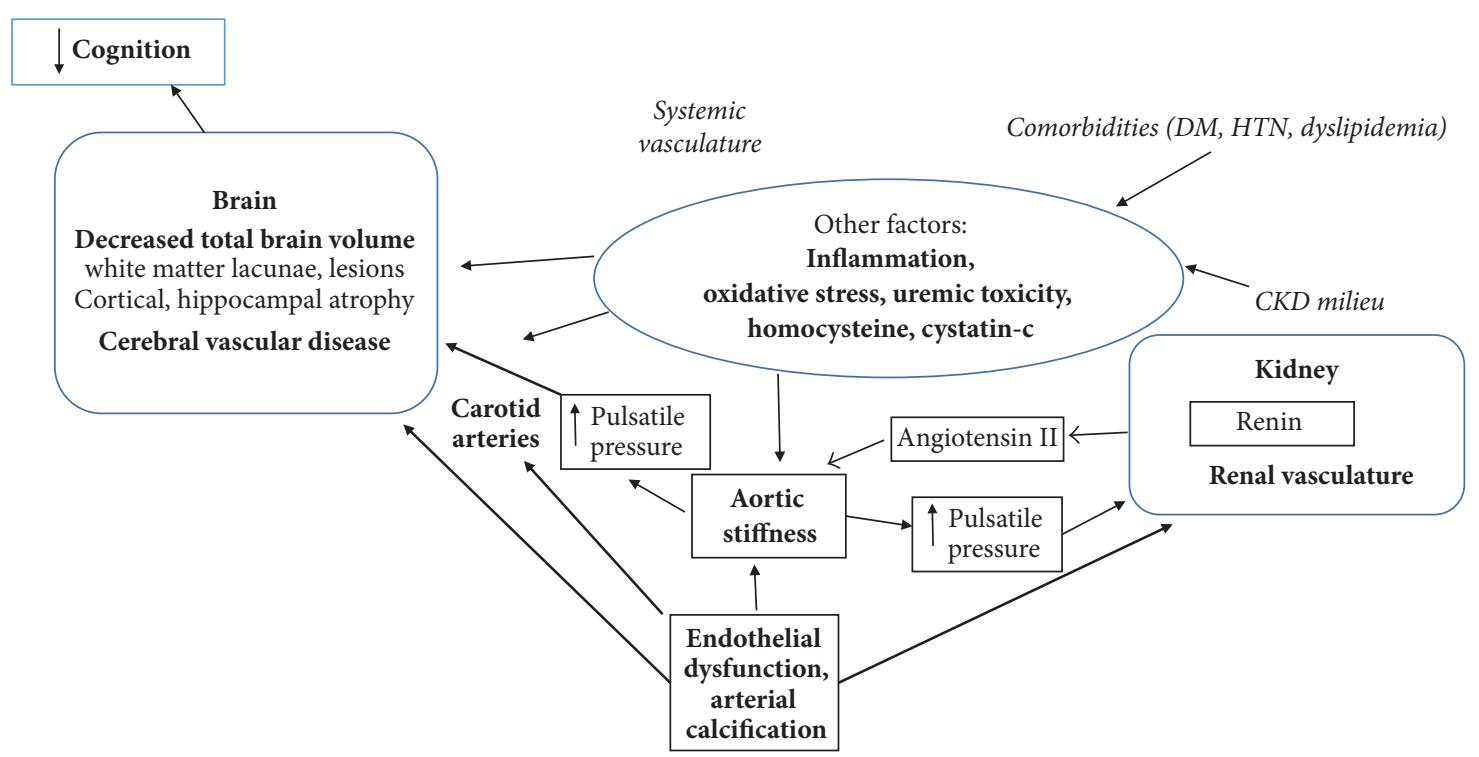

FIGURE 1: Systemic and cerebral vasculature and cognition in CKD.

cognitive impairment, including the dialysis procedure itself. The dialysis procedure predisposes patients to potential risk factors for cognitive impairment and cerebrovascular disease including volume and electrolyte fluctuations, cerebral edema and hypoperfusion, and excessive cytokine release [60]. Interestingly, the frequency of hypotensive episodes during dialysis has been associated with cerebral atrophy and lacunae frequency, while microembolisms may contribute to the burden of both large and small vessel cerebrovascular disease although much research is needed in this area $[77,78]$. Moreover, secondary and recurrent delirium (often related to hypoperfusion) and encephalopathy (i.e., untreated renal failure related neurotoxicity) appear to be associated with the development of cognitive impairment [79]. Finally, we recognize that anemia and derangements in serum vitamin $\mathrm{D}$ levels also contribute to the CKD milieu and potentially cognitive decline. In cross-sectional studies, anemia has been found to be associated with cognition in ESRD; however, in a longitudinal study, anemia was not an independent predictor of cognitive decline in elderly patients with CKD [80]. In terms of vitamin $\mathrm{D}$, a review by Cheng et al. (2016) notes that reduced levels of $25(\mathrm{OH})$-vitamin $\mathrm{D}$ may be contributing to cognitive impairment in CKD [81]. Clinical trials are needed to investigate the effect of vitamin $\mathrm{D}$ supplementation on cognitive outcomes.

\section{The Cerebrovascular-Renal Axis (Figure 1)}

The accelerated cognitive decline in older CKD patients appears to be due, in part, to the CKD disease process itself, which creates a toxic vascular and metabolic milieu that consists of chronic inflammation, oxidative stress, uremia, and systemic vascular endothelial dysfunction [82-88]. This toxic internal vascular and metabolic milieu is postulated to cause vascular dysfunction related impairment of the white matter that is superimposed on neurodegenerative damage caused by homocysteine, uremic toxins, creatinine, and cystatin $\mathrm{C}$ $[62,89]$. Homocysteine appears to be an especially strong risk factor for stroke in CKD patients via a direct neurotoxic effect, initiation of systemic inflammation, and endothelial dysfunction [90-94]. The increase in homocysteine is probably due to reduced renal clearance. Unfortunately, interventions with folate to reduce homocysteine levels have thus far been conflicting and disappointing [95-97]. Patients with CKD have increased levels of oxidative stress, caused by uremia, production of reactive oxygen species via physiological pathways (e.g., impaired/damaged/malfunctioning mitochondria), and an inability to produce adequate antioxidative enzymes $[98,99]$. These changes all contribute to a vascular milieu that consists of systemic inflammation, high levels of oxidative stress, and endothelial dysfunction that is unique to the CKD patient and creates a vascular pathway to cognitive decline.

\section{Vascular Mechanisms Related to Cognitive Decline in CKD}

Patients with CKD are at an increased risk for vascular disease-related cognitive impairment rather than Alzheimer's disease per se as described above. Vascular calcification in advanced stages of CKD, possibly including intracranial calcification, could be influencing cognition. Interestingly, there appears to be a significant influence of chronic hypertension on the progression of cognitive decline. This may be related to the high volume of blood flow and pressure that the brain and the kidney are exposed to.

The association between systemic arterial stiffness and cognitive performance has been established in cross-sectional studies [100]. More recently, Pase et al. (2016) studied the Framingham Offspring cohort and found that aortic stiffness predicts incident mild cognitive impairment and incident dementia in nondiabetic patients over 10 years [101]. Apart 
from aortic (central arterial) stiffness, stiffness in arteries in close proximity to the brain may need to be considered. One study reports that, in swines, carotid artery stiffness seems to be associated with impaired memory [102]. Additionally, although intracranial artery stiffness is even more challenging to measure, it may also be associated with cognitive decline [103].

Hypertension is associated with changes in brain tissue and cerebral vasculature. For example, mean arterial pressure was associated with white matter hyperintensity volume in the Framingham Offspring cohort, even in the absence of associations between changes in brain tissue and tonometry measures (such as arterial stiffness or central pulse pressure) [104]. Importantly, increased duration of hypertension is an important contributor to cognitive outcomes. Midlife hypertension has a significant impact on long-term cognitive impairment, as reviewed by Iadecola et al. (2016) [105]. Although some studies have shown a relationship between elevated blood pressure and cognitive impairment in the absence of a stroke, whether intensive hypertension control results in prevention or reversal of cognitive outcomes is unclear [106]. Upcoming results from the SPRINT-MIND trial (Systolic Blood Pressure Intervention Trial, Memory and Cognition in Decreased Hypertension) may address some of these unanswered questions.

The Strain Vessel Hypothesis states that "strain vessels" found in vital organs play a protective role [107]. Strain vessels help maintain a pressure gradient between the larger arteries and capillaries. High-pressure flow from large arteries, in addition to low resistance to flow in small vessels in vital organs, causes subsequent damage to vessels exposed to high pulsatility [108]. In the brain, small perforating arteries are exposed to high pressure; cerebral hemorrhage and infarction occur frequently in these small arteries [107, 109]. As decreasing kidney function is associated with arterial stiffness [110] and high blood pressure, patients with CKD are likely to have their blood vessels exposed to high pulsatility flow. Therefore, CKD patients are at high risk of developing injury to cerebral vasculature. The latter, in turn, would impact cognitive function.

It is challenging for drugs to influence the aorta and large arteries; and thus interventions may target other conduit arteries to reduce wave reflection. Although drugs such as angiotensin-converting enzyme inhibitors and calcium channel blockers seem to be beneficial in hypertensive elderly individuals, blood pressure levels that are optimal for cognitive function are yet to be identified [106]. Apart from medications, regular exercise may be employed to target this phenomenon.

\section{Exercise as a Potential Therapeutic Approach}

Higher levels of physical activity and cardiorespiratory fitness levels are associated with increased levels of cognitive function in healthy individuals. Exercise appears to prevent cerebral atrophy or even increase hippocampal volume in the general population $[111,112]$. It is conjectured that these observations are related to an increase in brain-derived neurotrophic factor and an exercise-induced increase in angiogenesis, neurogenesis, and synaptogenesis. It is conceivable that physical activity and fitness levels are related to cognitive function in patients with kidney disease, but there have been a minimal number of studies in this area and the results are inconsistent. Patients on hemodialysis with the highest self-reported activity levels had the highest cognitive scores in one study [113]. Conversely, one smaller study found no association between maximal oxygen consumption and scores on the MMSE in patients on hemodialysis [114]. Several exercise intervention studies have shown promise in improving cognition in healthy elderly participants with and without MCI [111, 115-126], whereas others have reported no improvement [127-130]. Regular exercise and higher fitness levels in non-CKD patients with and without cognitive impairment have been associated with improved cognitive function, white matter integrity, and hippocampal volume suggesting a possible neuroprotective effect of exercise [111, 113,115-125]. This is conjectured to be due to an improvement in vascular function-related increases in cerebral blood flow [116]. It is therefore plausible that exercise training may improve the vascular milieu and thereby contribute to improved cognitive function in patients with $\mathrm{CKD}$. However, the impact of exercise on cognitive function in the CKD population is currently unknown. Only one study has reported on cognitive function following exercise training in the dialysis population. Martins et al., 2011, reported an improvement in cognitive function measured via the MMSE following exercise training [131]. Unfortunately, this study was not randomized and the MMSE is not a sensitive measure for change in global cognition, which limits any conclusions. Studies investigating the impact of exercise on cognition in CKD patients are needed. Exercise training appears to improve the vascular milieu in patients with CKD by reducing systemic inflammation and oxidative stress, arterial stiffness, and improving vascular function [132]. However, not all studies have shown improvements in these vascular risk factors, likely due to differences in sample characteristics, exercise program, and outcome measures. Moreover, exercise training may reduce traditional risk factors for cerebrovascular disease such as blood pressure and lipid profile although it should be noted that randomized controlled trials are scarce in patients with CKD and most data are based on secondary analyses from smaller trials. Exercise training is also known to improve glucose control in diabetic patients and may reduce homocysteine levels. Importantly, the pleiotropic effect of exercise provides additional benefits that are important to patients with CKD including improved quality of life, improved physical function, and reduced risk of frailty. Moreover, higher levels of physical activity have been associated with reduced risk of initiation of renal replacement therapy and higher survival rate in patients with CKD stages 3-5 [133]. Finally, emerging studies suggest that there is an independent association between prolonged sedentary time and kidney function decline, whereas higher levels of physical activity are associated with reduced levels of creatinine and lower risk of kidney impairment $[134,135]$. Thus, it is plausible that exercise may affect renal function itself and thereby provide a protective effect. Despite lack of 
data on the impact of exercise on cognitive function, it is prudent for clinicians to recommend that patients with $\mathrm{CKD}$ consider initiating an exercise program and increase their daily physical activity levels to gain the mental and physical health benefits of exercise.

\section{Summary and Conclusions}

Cognitive impairment is common in patients with CKD and negatively affects health-related quality of life and other health-related outcomes. It is imperative that clinicians recognize the value of early screening for cognitive impairment and initiate preventive and treatment measures. Importantly, the decline in cognitive function appears to be multifaceted with a major involvement of vascular dysfunction in a unique CKD metabolic milieu that predisposes patients to an accelerated cognitive decline. Multidisciplinary healthcare teams are needed to provide psychosocial support and patient education on essential topics such as control of blood pressure, risks of polypharmacy, and other individualized self-care practices. Current research investigating exerciseinduced improvement in cognition in non-CKD population is promising, but there are conflicting reports in the literature. As exercise training may be a plausible adjunctive therapeutic approach to improve cognitive outcomes and quality of life in patients with CKD, further research should focus on exercise as a promising approach that may retard the progression of cognitive impairment in CKD.

\section{Conflicts of Interest}

The authors declare that they have no conflicts of interest.

\section{References}

[1] J. Coresh, E. Selvin, L. A. Stevens et al., "Prevalence of chronic kidney disease in the United States," Journal of the American Medical Association, vol. 298, no. 17, pp. 2038-2047, 2007.

[2] U.S. Renal Data System, "USRDS 2013 annual data report," Atlas of Chronic Kidney Disease \& End Stage Renal Disease in the United States, 2013.

[3] C. E. Rodriguez-Angarita, R. M. Sanabria-Arenas, J. D. VargasJaramillo, and I. Ronderos-Botero, "Cognitive impairment and depression in a population of patients with chronic kidney disease in colombia: a prevalence study," Canadian Journal of Kidney Health and Disease, vol. 3, article 26, 2016.

[4] M. K. Tamura, K. Yaffe, C.-Y. Hsu et al., "Cognitive Impairment and Progression of CKD," American Journal of Kidney Diseases, vol. 68, no. 1, pp. 77-83, 2016.

[5] M. Kurella Tamura, V. Wadley, K. Yaffe et al., "Kidney function and cognitive impairment in US adults: the reasons for geographic and racial differences in stroke (REGARDS) study," American Journal of Kidney Diseases, vol. 52, no. 2, pp. 227-234, 2008.

[6] M. Kurella, G. M. Chertow, J. Luan, and K. Yaffe, "Cognitive impairment in chronic kidney disease," Journal of the American Geriatrics Society, vol. 52, no. 11, pp. 1863-1869, 2004.

[7] M. Kurella, G. M. Chertow, L. F. Fried et al., "Chronic kidney disease and cognitive impairment in the elderly: the health, aging, and body composition study," Journal of the American Society of Nephrology, vol. 16, no. 7, pp. 2127-2133, 2005.

[8] K. Yaffe, L. Ackerson, M. K. Tamura et al., "Chronic kidney disease and cognitive function in older adults: findings from the chronic renal insufficiency cohort cognitive study," Journal of the American Geriatrics Society, vol. 58, no. 2, pp. 338-345, 2010.

[9] A. M. Murray, D. E. Tupper, D. S. Knopman et al., "Cognitive impairment in hemodialysis patients is common," Neurology, vol. 67, no. 2, pp. 216-223, 2006.

[10] A. Phinney, "Living with dementia from the patient's perspective," Journal of Gerontological Nursing, vol. 24, no. 6, pp. 8-15, 1998.

[11] E. Steeman, I. L. Abraham, and J. Godderis, "Risk profiles for institutionalization in a cohort of elderly people with dementia or depression," Archives of Psychiatric Nursing, vol. 11, no. 6, pp. 295-303, 1997.

[12] S. Andrieu, E. Reynish, F. Nourhashemi et al., "Predictive factors of acute hospitalization in 134 patients with Alzheimer's disease: a one year prospective study," International Journal of Geriatric Psychiatry, vol. 17, no. 5, p. 422, 2002.

[13] R. Newcomer, K. E. Covinsky, T. Clay, and K. Yaffe, "Predicting 12-month mortality for persons with dementia," Journals of Gerontology-Series B Psychological Sciences and Social Sciences, vol. 58, no. 3, pp. S187-S198, 2003.

[14] J. H. Wlodarczyk, H. Brodaty, and G. Hawthorne, "The relationship between quality of life, mini-mental state examination, and the instrumental activities of daily living in patients with Alzheimer's disease," Archives of Gerontology and Geriatrics, vol. 39, no. 1, pp. 25-33, 2004.

[15] A. R. Sehgal, S. F. Grey, P. B. DeOreo, and P. J. Whitehouse, "Prevalence, recognition, and implications of mental impairment among hemodialysis patients," American Journal of Kidney Diseases, vol. 30, no. 1, pp. 41-49, 1997.

[16] D. E. Weiner and S. L. Seliger, "Cognitive and physical function in chronic kidney disease," Current Opinion in Nephrology and Hypertension, vol. 23, no. 3, pp. 291-297, 2014.

[17] E. P. Sorensen, M. J. Sarnak, H. Tighiouart et al., “The kidney disease quality of life cognitive function subscale and cognitive performance in maintenance hemodialysis patients," American Journal of Kidney Diseases, vol. 60, no. 3, pp. 417-426, 2012.

[18] B. A. Bremer, K. M. Wert, A. L. Durica, and A. Weaver, "Neuropsychological, physical, and psychosocial functioning of individuals with end-stage renal disease," Annals of Behavioral Medicine, vol. 19, no. 4, pp. 348-352, 1997.

[19] S. Jung, Y.-K. Lee, S. R. Choi, S.-H. Hwang, and J.-W. Noh, "Relationship between cognitive impairment and depression in dialysis patients," Yonsei Medical Journal, vol. 54, no. 6, pp. 14471453, 2013.

[20] L. Feng, K. B. Yap, and T. P. Ng, "Depressive symptoms in older adults with chronic kidney disease: mortality, quality of life outcomes, and correlates," American Journal of Geriatric Psychiatry, vol. 21, no. 6, pp. 570-579, 2013.

[21] M. A. McAdams-Demarco, J. Tan, M. L. Salter et al., "Frailty and cognitive function in incident hemodialysis patients," Clinical Journal of the American Society of Nephrology, vol. 10, no. 12, pp. 2181-2189, 2015.

[22] U.S. Renal Data System, USRDS 2006 Annual Data Report: Atlas of Chronic Kidney Disease \& End Stage Renal Disease in the United States, 2006. 
[23] M. F. Folstein, S. E. Folstein, and P. R. McHugh, “"Mini-mental state. A practical method for grading the cognitive state of patients for the clinician," Journal of Psychiatric Research, vol. 12, no. 3, pp. 189-198, 1975.

[24] D. S. Knopman, B. F. Boeve, and R. C. Petersen, "Essentials of the proper diagnoses of mild cognitive impairment, dementia, and major subtypes of dementia," Mayo Clinic Proceedings, vol. 78, no. 10, pp. 1290-1308, 2003.

[25] R. C. Petersen, R. Doody, A. Kurz et al., "Current concepts in mild cognitive impairment," Archives of Neurology, vol. 58, no. 12, pp. 1985-1992, 2001.

[26] R. C. Petersen, "Mild cognitive impairment as a diagnostic entity," Journal of Internal Medicine, vol. 256, no. 3, pp. 183-194, 2004.

[27] American Psychiatric Associtation, Diagnostic and Statistical Manual of Mental Disorders (DSM-IV), American Psychiatric Association, Washington, DC, USA, 4th edition, 1994.

[28] M. Bossola, M. Antocicco, E. Di Stasio et al., "Mini Mental State Examination over time in chronic hemodialysis patients," Journal of Psychosomatic Research, vol. 71, no. 1, pp. 50-54, 2011.

[29] A. S. Buchman, D. Tanne, P. A. Boyle, R. C. Shah, S. E. Leurgans, and D. A. Bennett, "Kidney function is associated with the rate of cognitive decline in the elderly," Neurology, vol. 73, no. 12, pp. 920-927, 2009.

[30] T. Etgen, D. Sander, M. Chonchol et al., "Chronic kidney disease is associated with incident cognitive impairment in the elderly: The INVADE study," Nephrology Dialysis Transplantation, vol. 24, no. 10, pp. 3144-3150, 2009.

[31] M. Kurella, D. L. Mapes, F. K. Port, and G. M. Chertow, "Correlates and outcomes of dementia among dialysis patients: the dialysis outcomes and practice patterns study," Nephrology Dialysis Transplantation, vol. 21, no. 9, pp. 2543-2548, 2006.

[32] A. M. Murray, J. I. Barzilay, J. F. Lovato et al., "Biomarkers of renal function and cognitive impairment in patients with diabetes," Diabetes Care, vol. 34, no. 8, pp. 1827-1832, 2011.

[33] K. Yaffe, K. Lindquist, M. G. Shlipak et al., "Cystatin C as a marker of cognitive function in elders: findings from the Health ABC Study," Annals of Neurology, vol. 63, no. 6, pp. 798-802, 2008.

[34] M. K. Georgakis, N. G. Dimitriou, M. A. Karalexi et al., "Albuminuria in Association with Cognitive Function and Dementia: A Systematic Review and Meta-Analysis," Journal of the American Geriatrics Society, 2017.

[35] M. Kurella, K. Yaffe, M. G. Shlipak, N. K. Wenger, and G. M. Chertow, "Chronic kidney disease and cognitive impairment in menopausal women," American Journal of Kidney Diseases, vol. 45, no. 1, pp. 66-76, 2005.

[36] I. Sajjad, F. Grodstein, J. H. Kang, G. C. Curhan, and J. Lin, "Kidney dysfunction and cognitive decline in women," Clinical Journal of the American Society of Nephrology, vol. 7, no. 3, pp. 437-443, 2012.

[37] J. A. Passer, "Cerebral atrophy in end-stage uremia," Proceedings of the Clinical Dialysis and Transplant Forum, vol. 7, pp. 91-94, 1977.

[38] T. Kamata, A. Hishida, T. Takita et al., "Morphologic abnormalities in the brain of chronically hemodialyzed patients without cerebrovascular disease," American Journal of Nephrology, vol. 20, no. 1, pp. 27-31, 2000.

[39] G. M. Savazzi, F. Cusmano, and S. Musini, "Cerebral imaging changes in patients with chronic renal failure treated conservatively or in hemodialysis," Nephron, vol. 89, no. 1, pp. 31-36, 2001.
[40] A.-H. Cho, S. B. Lee, S. J. Han, Y.-M. Shon, D.-W. Yang, and B. S. Kim, "Impaired kidney function and cerebral microbleeds in patients with acute ischemic stroke," Neurology, vol. 73, no. 20, pp. 1645-1648, 2009.

[41] G. Fazekas, F. Fazekas, R. Schmidt, P. Kapeller, H. Offenbacher, and G. J. Krejs, "Brain MRI findings and cognitive impairment in patients undergoing chronic hemodialysis treatment," Journal of the Neurological Sciences, vol. 134, no. 1-2, pp. 83-88, 1995.

[42] H. Shima, E. Ishimura, T. Naganuma et al., "Cerebral microbleeds in predialysis patients with chronic kidney disease," Nephrology Dialysis Transplantation, vol. 25, no. 5, pp. 15541559,2010

[43] M. A. Ikram, M. W. Vernooij, A. Hofman, W. J. Niessen, A. Van Der Lugt, and M. M. B. Breteler, "Kidney function is related to cerebral small vessel disease," Stroke, vol. 39, no. 1, pp. 55-61, 2008.

[44] M. Wada, H. Nagasawa, C. Iseki et al., "Cerebral small vessel disease and chronic kidney disease (CKD): results of a crosssectional study in community-based Japanese elderly," Journal of the Neurological Sciences, vol. 272, no. 1-2, pp. 36-42, 2008.

[45] S. Sedaghat, L. G. M. Cremers, M. De Groot et al., "Kidney function and microstructural integrity of brain white matter," Neurology, vol. 85, no. 2, pp. 154-161, 2015.

[46] A. M. Tuladhar, A. G. van Norden, K. F. de Laat et al., "White matter integrity in small vessel disease is related to cognition," NeuroImage: Clinical, vol. 7, pp. 518-524, 2015.

[47] H. J. Chen, L. J. Zhang, and G. M. Lu, "Multimodality MRI findings in patients with end-stage renal disease," BioMed Research International, vol. 2015, Article ID 697402, 12 pages, 2015.

[48] L. E. Oberlin, T. D. Verstynen, A. Z. Burzynska et al., "White matter microstructure mediates the relationship between cardiorespiratory fitness and spatial working memory in older adults," NeuroImage, vol. 131, pp. 91-101, 2016.

[49] M. W. Vernooij, M. A. Ikram, H. A. Vrooman et al., "White Matter microstructural integrity and cognitive function in a general elderly population," Archives of General Psychiatry, vol. 66, no. 5, pp. 545-553, 2009.

[50] M.-C. Chou, T.-J. Hsieh, Y.-L. Lin et al., "Widespread white matter alterations in patients with end-stage renal disease: a voxelwise diffusion tensor imaging study," American Journal of Neuroradiology, vol. 34, no. 10, pp. 1945-1951, 2013.

[51] X. Kong, J.-Q. Wen, R.-F. Qi et al., "Diffuse interstitial brain edema in patients with end-stage renal disease undergoing hemodialysis: A Tract-Based Spatial Statistics Study," Medicine, vol. 93, no. 28, article e313, 2014.

[52] R. Zhang, K. Liu, L. Yang et al., "Reduced white matter integrity and cognitive deficits in maintenance hemodialysis ESRD patients: a diffusion-tensor study," European Radiology, vol. 25, no. 3, pp. 661-668, 2014.

[53] H. S. Kim, J. W. Park, D. S. Bai et al., "Diffusion tensor imaging findings in neurologically asymptomatic patients with end stage renal disease," NeuroRehabilitation, vol. 29, no. 1, pp. 111-116, 2011.

[54] M. Lamar, X. J. Zhou, R. A. Charlton, D. Dean, D. Little, and S. C. Deoni, "In vivo quantification of white matter microstructure for use in aging: a focus on two emerging techniques," American Journal of Geriatric Psychiatry, vol. 22, no. 2, pp. 111-121, 2014.

[55] L. J. Zhang, J. Wen, X. Liang et al., "Brain default mode network changes after renal transplantation: a diffusion-tensor imaging and resting-state functional MR imaging study," Radiology, vol. 278, no. 2, pp. 485-495, 2016. 
[56] S. Lavi, D. Gaitini, V. Milloul, and G. Jacob, "Impaired cerebral $\mathrm{CO}_{2}$ vasoreactivity: association with endothelial dysfunction," American Journal of Physiology - Heart and Circulatory Physiology, vol. 291, no. 4, pp. H1856-H1861, 2006.

[57] S. Sedaghat, M. W. Vernooij, E. Loehrer et al., "Kidney function and cerebral blood flow: The Rotterdam Study," Journal of the American Society of Nephrology, vol. 27, no. 3, pp. 715-721, 2016.

[58] J. Radić, D. Ljutić, M. Radić, V. Kovačić, K. Dodig-Ćurković, and M. Šain, "Kidney transplantation improves cognitive and psychomotor functions in adult hemodialysis patients," American Journal of Nephrology, vol. 34, no. 5, pp. 399-406, 2011.

[59] W. L. Lau, B. N. Huisa, and M. Fisher, "the cerebrovascularchronic kidney disease connection: perspectives and mechanisms," Translational Stroke Research, vol. 8, no. 1, pp. 67-76, 2017.

[60] R. Lu, M. C. Kiernan, A. Murray, M. H. Rosner, and C. Ronco, "Kidney-brain crosstalk in the acute and chronic setting," Nature Reviews Nephrology, vol. 11, no. 12, pp. 707-719, 2015.

[61] M. Madero, A. Gul, and M. J. Sarnak, "Cognitive function in chronic kidney disease," Seminars in Dialysis, vol. 21, no. 1, pp. 29-37, 2008.

[62] J.-M. Bugnicourt, O. Godefroy, J.-M. Chillon, G. Choukroun, and Z. A. Massy, "Cognitive disorders and dementia in CKD: the neglected kidney-brain axis," Journal of the American Society of Nephrology, vol. 24, no. 3, pp. 353-363, 2013.

[63] M. Kobayashi, N. Hirawa, K. Yatsu et al., "Relationship between silent brain infarction and chronic kidney disease," Nephrology Dialysis Transplantation, vol. 24, no. 1, pp. 201-207, 2009.

[64] B. Ovbiagele, J. J. Wing, R. S. Menon et al., "Association of chronic kidney disease with cerebral microbleeds in patients with primary intracerebral hemorrhage," Stroke, vol. 44, no. 9, pp. 2409-2413, 2013.

[65] A. M. Murray, "Cognitive impairment in the aging dialysis and chronic kidney disease populations: an occult burden," Advances in Chronic Kidney Disease, vol. 15, no. 2, pp. 123-132, 2008.

[66] H.-H. Wang, S.-Y. Hung, J.-M. Sung, K.-Y. Hung, and J.-D. Wang, "Risk of stroke in long-term dialysis patients compared with the general population," American Journal of Kidney Diseases, vol. 63, no. 4, pp. 604-611, 2014.

[67] R. N. Foley, D. T. Gilbertson, T. Murray, and A. J. Collins, "Long interdialytic interval and mortality among patients receiving hemodialysis," The New England Journal of Medicine, vol. 365, no. 12, pp. 1099-1107, 2011.

[68] C. Helmer, B. Stengel, M. Metzger et al., "Chronic kidney disease, cognitive decline, and incident dementia: the 3C Study," Neurology, vol. 77, no. 23, pp. 2043-2051, 2011.

[69] C. Gaxatte, M. Daroux, J. Bloch, F. Puisieux, V. Deramecourt, and E. Boulanger, "Cognitive impairment and chronic kidney disease: which links?” Nephrologie et Therapeutique, vol. 7, no. 1, pp. 10-17, 2011.

[70] T. Umemura, T. Kawamura, H. Umegaki et al., "Association of chronic kidney disease and cerebral small vessel disease with cognitive impairment in elderly patients with type 2 diabetes," Dementia and Geriatric Cognitive Disorders Extra, vol. 3, no. 1, pp. 212-222, 2013.

[71] A. F. Perna, D. Ingrosso, E. Violetti et al., "Hyperhomocysteinemia in uremia-a red flag in a disrupted circuit," Seminars in Dialysis, vol. 22, no. 4, pp. 351-356, 2009.

[72] S. L. Seliger, D. L. Gillen, D. Tirschwell, H. Wasse, B. R. Kestenbaum, and C. O. Stehman-Breen, "Risk factors for incident stroke among patients with end-stage renal disease," Journal of the American Society of Nephrology, vol. 14, no. 10, pp. 26232631, 2003.

[73] J. B. Wetmore, E. F. Ellerbeck, J. D. Mahnken et al., "Atrial fibrillation and risk of stroke in dialysis patients," Annals of Epidemiology, vol. 23, no. 3, pp. 112-118, 2013.

[74] P. B. Gorelick, J. Brody, D. Cohen et al., "Risk factors for dementia associated with multiple cerebral infarcts. A casecontrol analysis in predominantly African-American hospitalbased patients," Archives of Neurology, vol. 50, no. 7, pp. 714-720, 1993.

[75] M. K. Tamura, D. Xie, K. Yaffe et al., "Vascular risk factors and cognitive impairment in chronic kidney disease: the Chronic Renal Insufficiency Cohort (CRIC) study," Clinical Journal of the American Society of Nephrology, vol. 6, no. 2, pp. 248-256, 2011.

[76] E. A. Iliescu, H. Coo, M. H. McMurray et al., "Quality of sleep and health-related quality of life in haemodialysis patients," Nephrology Dialysis Transplantation, vol. 18, no. 1, pp. 126-132, 2003.

[77] D. W. Droste, K. Kühne, R. M. Schaefer, and E. B. Ringelstein, "Detection of microemboli in the subclavian vein of patients undergoing haemodialysis and haemodiafiltration using pulsed Doppler ultrasound," Nephrology Dialysis Transplantation, vol. 17, no. 3, pp. 462-466, 2002.

[78] T. Mizumasa, H. Hirakata, T. Yoshimitsu et al., "Dialysis-related hypotension as a cause of progressive frontal lobe atrophy in chronic hemodialysis patients: a 3-year prospective study," Nephron Clinical Practice, vol. 97, no. 1, pp. c23-c30, 2004.

[79] R. Brouns and P. P. De Deyn, "Neurological complications in renal failure: a review," Clinical Neurology and Neurosurgery, vol. 107, no. 1, pp. 1-16, 2004.

[80] M. Kurella Tamura, E. Vittinghoff, J. Yang et al., "Anemia and risk for cognitive decline in chronic kidney disease," $B M C$ Nephrology, vol. 17, no. 1, article 226, 2016.

[81] Z. Cheng, J. Lin, and Q. Qian, "Role of vitamin D in cognitive function in chronic kidney disease," Nutrients, vol. 8, no. 5, article 291, 2016.

[82] J. Himmelfarb, "Uremic toxicity, oxidative stress, and hemodialysis as renal replacement therapy," Seminars in Dialysis, vol. 22, no. 6, pp. 636-643, 2009.

[83] S. L. Seliger and W. T. Longstreth Jr., "Lessons about brain vascular disease from another pulsating organ, the kidney," Stroke, vol. 39, no. 1, pp. 5-6, 2008.

[84] J. M. Wardlaw, P. A. G. Sandercock, M. S. Dennis, and J. Starr, "Is breakdown of the blood-brain barrier responsible for lacunar stroke, leukoaraiosis, and dementia?" Stroke, vol. 34, no. 3, pp. 806-811, 2003.

[85] H. Kalimo, "Does chronic brain edema explain the consequences of cerebral small-vessel disease?" Stroke, vol. 34, no. 3, pp. 806-812, 2003.

[86] D. E. Weiner, K. Bartolomei, T. Scott et al., "Albuminuria, cognitive functioning, and white matter hyperintensities in homebound elders," American Journal of Kidney Diseases, vol. 53, no. 3, pp. 438-447, 2009.

[87] C. Berr, B. Balansard, J. Arnaud, A. M. Roussel, and A. Alperovitch, "Cognitive decline is associated with systemic oxidative stress: the EVA study. etude du vieillissement arteriel," Journal of the American Geriatrics Society, vol. 48, no. 10, pp. 1285-1291, 2000.

[88] T. A. Ikizler, J. D. Morrow, L. J. Roberts et al., "Plasma F2-isoprostane levels are elevated in chronic hemodialysis patients," Clinical Nephrology, vol. 58, no. 3, pp. 190-197, 2002. 
[89] P. P. De Deyn, R. Vanholder, S. Eloot, and G. Glorieux, "Guanidino compounds as uremic (neuro)toxins," Seminars in Dialysis, vol. 22, no. 4, pp. 340-345, 2009.

[90] F. Anan, N. Takahashi, T. Shimomura et al., "Hyperhomocysteinemia is a significant risk factor for silent cerebral infarction in patients with chronic renal failure undergoing hemodialysis," Metabolism, vol. 55, no. 5, pp. 656-661, 2006.

[91] K. Faßbender, O. Mielke, T. Bertsch, B. Nafe, S. Fröschen, and M. Hennerici, "Homocysteine in cerebral macroangiography and microangiopathy," The Lancet, vol. 353, no. 9164, pp. 15861587, 1999.

[92] S. A. Lipton, W.-K. Kim, Y.-B. Choi et al., "Neurotoxicity associated with dual actions of homocysteine at the N-methylD-aspartate receptor," Proceedings of the National Academy of Sciences of the United States of America, vol. 94, no. 11, pp. 59235928, 1997.

[93] S. Seshadri, P. A. Wolf, A. S. Beiser et al., "Association of plasma total homocysteine levels with subclinical brain injury: cerebral volumes, white matter hyperintensity, and silent brain infarcts at volumetric magnetic resonance imaging in the Framingham Offspring Study," Archives of Neurology, vol. 65, no. 5, pp. 642649, 2008.

[94] C. B. Wright, M. C. Paik, T. R. Brown et al., "Total homocysteine is associated with white matter hyperintensity volume: the Northern Manhattan study," Stroke, vol. 36, no. 6, pp. 1207-1211, 2005.

[95] M. Lee, K.-S. Hong, S.-C. Chang, and J. L. Saver, "Efficacy of homocysteine-lowering therapy with folic acid in stroke prevention: a meta-analysis," Stroke, vol. 41, no. 6, pp. 1205-1212, 2010.

[96] C. A. De Jager, A. Oulhaj, R. Jacoby, H. Refsum, and A. D. Smith, "Cognitive and clinical outcomes of homocysteine-lowering Bvitamin treatment in mild cognitive impairment: a randomized controlled trial," International Journal of Geriatric Psychiatry, vol. 27, no. 6, pp. 592-600, 2012.

[97] T. Kwok, J. Lee, C. B. Law et al., "A randomized placebo controlled trial of homocysteine lowering to reduce cognitive decline in older demented people," Clinical Nutrition, vol. 30, no. 3, pp. 297-302, 2011.

[98] K. Fujisaki, K. Tsuruya, M. Yamato et al., "Cerebral oxidative stress induces spatial working memory dysfunction in uremic mice: neuroprotective effect of tempol," Nephrology Dialysis Transplantation, vol. 29, no. 3, pp. 529-538, 2014.

[99] D. Salisbury and U. Bronas, "Reactive oxygen and nitrogen species: impact on endothelial dysfunction," Nursing Research, vol. 64, no. 1, pp. 53-66, 2015.

[100] A. Zeki Al Hazzouri and K. Yaffe, "Arterial stiffness and cognitive function in the elderly," Journal of Alzheimer's Disease, vol. 42, supplement 4, pp. S503-S514, 2014.

[101] M. P. Pase, J. J. Himali, G. F. Mitchell et al., "Association of aortic stiffness with cognition and brain aging in young and middleaged adults: The Framingham Third Generation Cohort Study," Hypertension, vol. 67, no. 3, pp. 513-519, 2016.

[102] T. D. Olver, D. Klakotskaia, B. S. Ferguson et al., "Carotid artery vascular mechanics serve as biomarkers of cognitive dysfunction in aortic-banded miniature swine that can be treated with an exercise intervention," Journal of the American Heart Association, vol. 5, no. 5, Article ID e003248, 2016.

[103] X. Sun and T. Rundek, "Does increased arterial stiffness herald cognitive impairment?” Stroke, vol. 47, no. 9, pp. 2171-2172, 2016.
[104] C. W. Tsao, J. J. Himali, A. S. Beiser et al., "Association of arterial stiffness with progression of subclinical brain and cognitive disease," Neurology, vol. 86, no. 7, pp. 619-626, 2016.

[105] C. Iadecola, K. Yaffe, J. Biller et al., "Impact of hypertension on cognitive function: a scientific statement from the American Heart Association," Hypertension, vol. 68, no. 6, pp. e67-e94, 2016.

[106] M. Tadic, C. Cuspidi, and D. Hering, "Hypertension and cognitive dysfunction in elderly: blood pressure management for this global burden," BMC Cardiovascular Disorders, vol. 16, article 208, 2016.

[107] S. Ito, T. Nagasawa, M. Abe, and T. Mori, "Strain vessel hypothesis: a viewpoint for linkage of albuminuria and cerebrocardiovascular risk," Hypertension Research, vol. 32, no. 2, pp. 115-121, 2009.

[108] M. F. O’Rourke and M. E. Safar, "Relationship between aortic stiffening and microvascular disease in brain and kidney: cause and logic of therapy," Hypertension, vol. 46, no. 1, pp. 200-204, 2005.

[109] G. F. Mitchell, M. A. Van Buchem, S. Sigurdsson et al., "Arterial stiffness, pressure and flow pulsatility and brain structure and function: The Age, Gene/Environment Susceptibility-Reykjavik Study," Brain, vol. 134, no. 11, pp. 3398-3407, 2011.

[110] R. R. Townsend, N. J. Wimmer, J. A. Chirinos et al., "Aortic PWV in chronic kidney disease: a CRIC ancillary study," American Journal of Hypertension, vol. 23, no. 3, pp. 282-289, 2010.

[111] K. I. Erickson, M. W. Voss, R. S. Prakash et al., "Exercise training increases size of hippocampus and improves memory," Proceedings of the National Academy of Sciences of the United States of America, vol. 108, no. 7, pp. 3017-3022, 2011.

[112] J. Weuve, J. H. Kang, J. E. Manson, M. M. B. Breteler, J. H. Ware, and F. Grodstein, "Physical activity, including walking, and cognitive function in older women," Journal of the American Medical Association, vol. 292, no. 12, pp. 1454-1461, 2004.

[113] F. Stringuetta-Belik, F. G. Shiraishi, V. R. Oliveira e Silva et al., "Greater level of physical activity associated with better cognitive function in hemodialysis in end stage renal disease," Jornal Brasileiro de Nefrologia, vol. 34, no. 4, pp. 378-386, 2012.

[114] R.-L. Hsieh, W.-C. Lee, and C.-H. Chang, "Maximal cardiovascular fitness and its correlates in ambulatory hemodialysis patients," American Journal of Kidney Diseases, vol. 48, no. 1, pp. 21-27, 2006.

[115] J. E. Ahlskog, Y. E. Geda, N. R. Graff-Radford, and R. C. Petersen, "Physical exercise as a preventive or diseasemodifying treatment of dementia and brain aging," Mayo Clinic Proceedings, vol. 86, no. 9, pp. 876-884, 2011.

[116] S. M. Hayes, M. L. Alosco, and D. E. Forman, "The effects of aerobic exercise on cognitive and neural decline in aging and cardiovascular disease," Current Geriatrics Reports, vol. 3, no. 4, pp. 282-290, 2014.

[117] J. C. Smith, K. A. Nielson, J. L. Woodard et al., "Does physical activity influence semantic memory activation in amnestic mild cognitive impairment?” Psychiatry Research, vol. 193, no. 1, pp. 60-62, 2011.

[118] R. Ruscheweyh, C. Willemer, K. Krüger et al., "Physical activity and memory functions: an interventional study," Neurobiology of Aging, vol. 32, no. 7, pp. 1304-1319, 2011.

[119] L. D. Baker, L. L. Frank, K. Foster-Schubert et al., "Effects of aerobic exercise on mild cognitive impairment: a controlled trial," Archives of Neurology, vol. 67, no. 1, pp. 71-79, 2010. 
[120] L. S. Nagamatsu, A. Chan, J. C. Davis et al., "Physical activity improves verbal and spatial memory in older adults with probable mild cognitive impairment: a 6-month randomized controlled trial," Journal of Aging Research, vol. 2013, Article ID 861893, 10 pages, 2013.

[121] N. J. Kirk-Sanchez and E. L. McGough, "Physical exercise and cognitive performance in the elderly: current perspectives," Clinical Interventions in Aging, vol. 9, pp. 51-62, 2013.

[122] M. W. Voss, K. I. Erickson, R. S. Prakash et al., "Neurobiological markers of exercise-related brain plasticity in older adults," Brain, Behavior, and Immunity, vol. 28, pp. 90-99, 2013.

[123] S. J. Colcombe, A. F. Kramer, K. I. Erickson et al., "Cardiovascular fitness, cortical plasticity, and aging," Proceedings of the National Academy of Sciences of the United States of America, vol. 101, no. 9, pp. 3316-3321, 2004.

[124] D. E. Barnes, W. Santos-Modesitt, G. Poelke et al., "The mental activity and exercise (MAX) trial: a randomized controlled trial to enhance cognitive function in older adults," JAMA Internal Medicine, vol. 173, no. 9, pp. 797-804, 2013.

[125] N. T. Lautenschlager, K. L. Cox, L. Flicker et al., "Effect of physical activity on cognitive function in older adults at risk for Alzheimer disease: a randomized trial," JAMA, vol. 300, no. 9, pp. 1027-1037, 2008.

[126] P. J. Smith, J. A. Blumenthal, B. M. Hoffman et al., "Aerobic exercise and neurocognitive performance: a meta-analytic review of randomized controlled trials," Psychosomatic Medicine, vol. 72, no. 3, pp. 239-252, 2010.

[127] J. A. Blumenthal and D. J. Madden, "Effects of aerobic exercise training, age, and physical fitness on memory-search performance," Psychology and Aging, vol. 3, no. 3, pp. 280-285, 1988.

[128] D. Laurin, R. Verreault, J. Lindsay, K. MacPherson, and K. Rockwood, "Physical activity and risk of cognitive impairment and dementia in elderly persons," Archives of Neurology, vol. 58, no. 3, pp. 498-504, 2001.

[129] J. D. Williamson, M. Espeland, S. B. Kritchevsky et al., "Changes in cognitive function in a randomized trial of physical activity: results of the lifestyle interventions and independence for elders pilot study," Journals of Gerontology, Series A: Biological Sciences and Medical Sciences, vol. 64, no. 6, pp. 688-694, 2009.

[130] K. Okumiya, K. Matsubayashi, T. Wada, S. Kimura, Y. Doi, and T. Ozawa, "Effects of exercise on neurobehavioral function in community-dwelling older people more than 75 years of age," Journal of the American Geriatrics Society, vol. 44, no. 5, pp. 569$572,1996$.

[131] C. T. Martins, G. S. Ramos, S. A. Guaraldo, C. B. Uezima, J. P. Martins, and E. Ribeiro Junior, "Comparison of cognitive function between patients on chronic hemodialysis who carry out assisted physical activity and inactive ones," Jornal Brasileiro de Nefrologia, vol. 33, no. 1, pp. 27-30, 2011.

[132] U. G. Bronas, "Exercise training and reduction of cardiovascular disease risk factors in patients with chronic kidney disease," Advances in Chronic Kidney Disease, vol. 16, no. 6, pp. 449-458, 2009.

[133] I.-R. Chen, S.-M. Wang, C.-C. Liang et al., "Association of walking with survival and RRT among patients with CKD stages 3-5," Clinical Journal of the American Society of Nephrology, vol. 9, no. 7, pp. 1183-1189, 2014.

[134] V. Y. Guo, S. Brage, U. Ekelund, S. J. Griffin, and R. K. Simmons, "Objectively measured sedentary time, physical activity and kidney function in people with recently diagnosed Type 2 diabetes: a prospective cohort analysis," Diabetic Medicine, vol. 33, no. 9, pp. 1222-1229, 2016.
[135] S. Lee, H. Shimada, S. Lee et al., "Association between sedentary time and kidney function in community-dwelling elderly Japanese people," Geriatrics \& Gerontology International, 2016. 


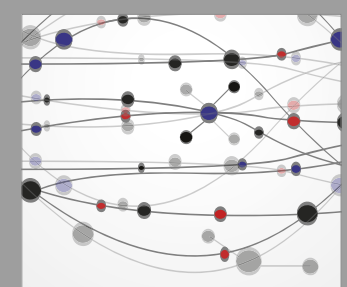

The Scientific World Journal
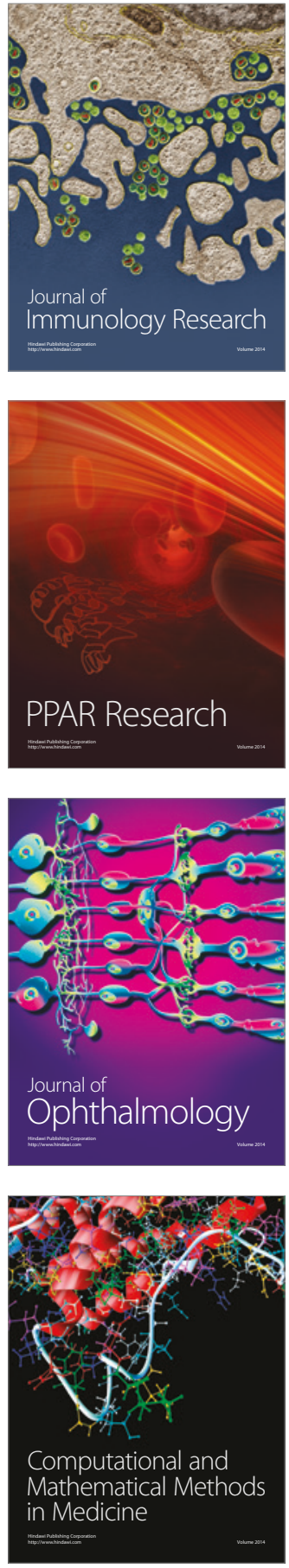

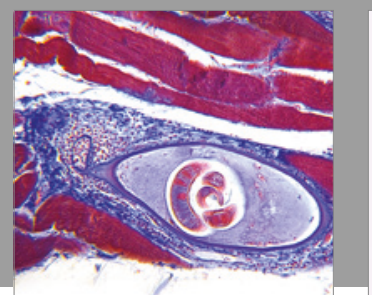

Gastroenterology Research and Practice
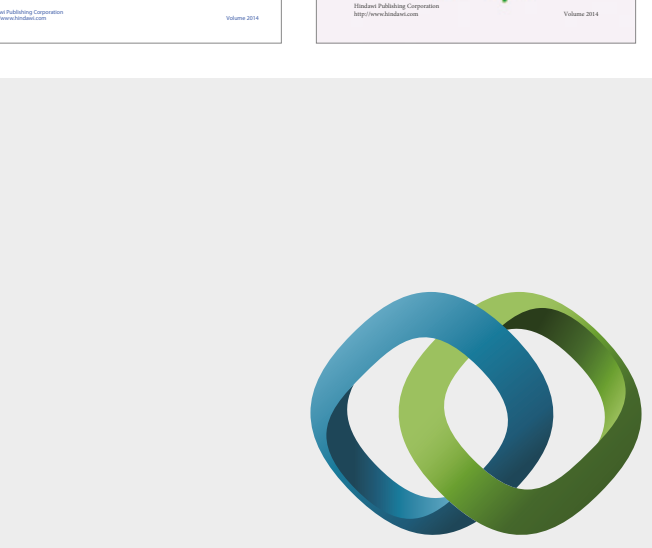

\section{Hindawi}

Submit your manuscripts at

https://www.hindawi.com
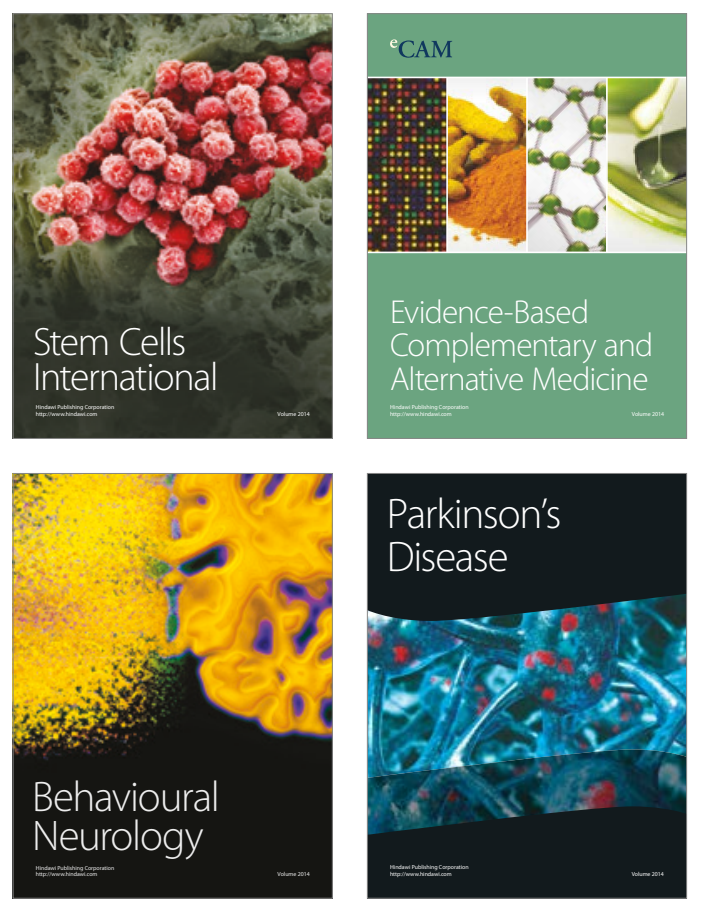
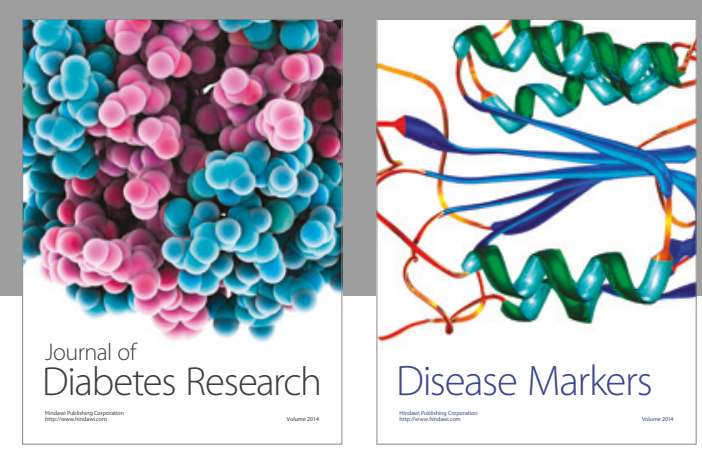

Disease Markers
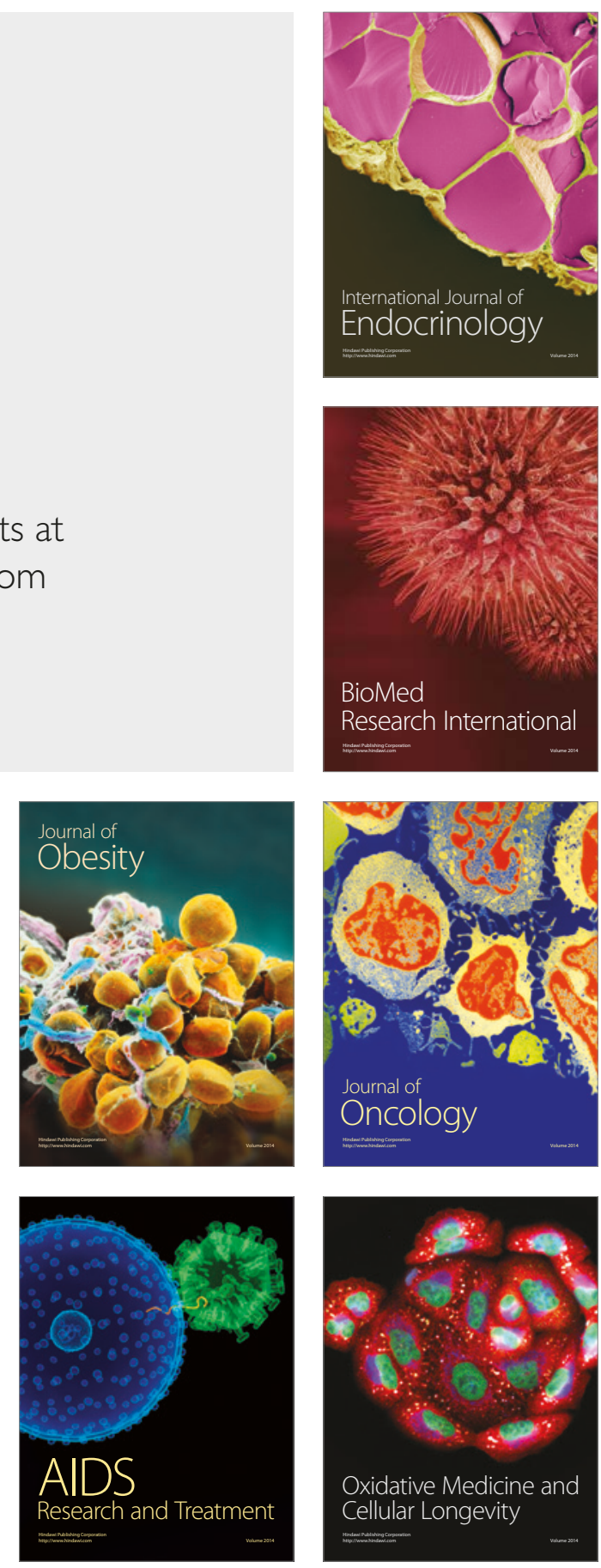